\title{
Symplectic Cohomologies on Phase Space
}

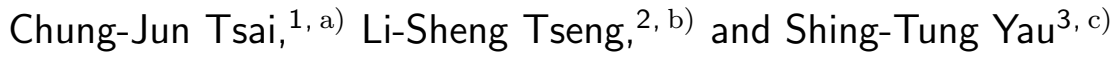

1) Mathematics Division, National Center for Theoretical Sciences (Taipei Office)

National Taiwan University, Taipei 1061\%, Taiwan

${ }^{2)}$ Department of Mathematics, University of California

Irvine, CA 92697 USA

3) Department of Mathematics, Harvard University

Cambridge, MA 02138 USA

(Dated: Friday $29^{\text {th }}$ June, 2012)

The phase space of a particle or a mechanical system contains an intrinsic symplectic structure, and hence, it is a symplectic manifold. Recently, new invariants for symplectic manifolds in terms of cohomologies of differential forms have been introduced by Tseng and Yau. Here, we discuss the physical motivation behind the new symplectic invariants and analyze these invariants for phase space, i.e. the non-compact cotangent bundle.

Dedicated to Elliott Lieb on the occasion of his 80th birthday.

a)Electronic mail: cjtsai@ntu.edu.tw

b)Electronic mail: lstseng@math.uci.edu

c)Electronic mail: yau@math.harvard.edu 


\section{INTRODUCTION}

In Hamiltonian mechanics, the description of a particle in motion is given by its position and momentum coordinates, $\left(x_{i}, p^{i}\right)$, which together constitute the variables of what is standardly called the phase space of the particle. Provided with a Hamiltonian function $H\left(x_{i}, p^{i}\right)$, the dependence of the position and momentum of the particle as a function of time is specified by the Hamilton equations of motion:

$$
\begin{aligned}
\frac{d}{d t} x_{i} & =\frac{\partial H}{\partial p^{i}}, \\
\frac{d}{d t} p^{i} & =-\frac{\partial H}{\partial x_{i}} .
\end{aligned}
$$

For a particle moving on a general manifold $M$, the position coordinates $\left\{x_{i}\right\}$ correspond to the local coordinates of a coordinate chart. In the overlap region between two coordinate neighborhoods, we can make a coordinate transformation to relate the two different coordinates, i.e. $x_{i}^{\prime}=x_{i}^{\prime}(q)$. The tangent vectors and the dual cotangent one-forms on $M$ transform under a coordinate transformation as

$$
\frac{\partial}{\partial x_{i}^{\prime}}=\frac{\partial x_{j}}{\partial x_{i}^{\prime}} \frac{\partial}{\partial x_{j}}, \quad d x_{i}^{\prime}=\frac{\partial x_{i}^{\prime}}{\partial x_{j}} d x_{j} .
$$

Moreover, by equation (I.2), the momentum coordinate $p^{i}$ must transform identically as that of the tangent vector $\partial / \partial x_{i}$. Hence, the momentum coordinates transform as

$$
p^{i}=\frac{\partial x_{j}}{\partial x_{i}^{\prime}} p^{j}
$$

With $\left(x_{i}, p^{i}\right)$ transforming as above under coordinate change, it is clear that there exists a global one-form on phase space called the tautological one-form

$$
\alpha=p^{i} d x_{i}^{\prime}=p^{i} d x_{i}
$$

From this point of view, $\left\{d x_{i}\right\}$ span a basis of cotangent one-forms and so $p^{i}$ can be considered as the fiber coordinates of the cotangent bundle $T^{*} M$. Hence, we have arrived at the wellknown fact that the classical phase space of a particle on $M$ is mathematically just $T^{*} M$. Now, taking the exterior derivative $d=d x_{i} \wedge \otimes \frac{\partial}{\partial x_{i}}$ of $\alpha$ in (I.3), we obtain a natural two-form on phase space

$$
\omega=-d \alpha=d x_{i} \wedge d p^{i}
$$


This two-form is non-degenerate and clearly vanishes when operated again by the exterior derivative operator $d$ (i.e. it is $d$-closed) and hence $\omega$ provides a natural symplectic structure on phase space, and therefore, $T^{*} M$ is a symplectic manifold.

In this paper, we would like to begin the exploration of symplectic invariants that arise from studying the differential forms on phase space, $X=T^{*} M$. The space of differential forms, denoted by $\Omega^{k}(X)$ for differential forms of degree $k$, encodes some of the simplest geometrical/topological data on manifolds. For instance, the $k$ th-de Rham cohomology $H^{k}(X)$ of a smooth manifold $X$, is the quotient vector space of differential $k$-forms given by

$$
H_{d R}^{k}(X)=\frac{\left\{A \in \Omega^{k}(X) \mid d A=0\right\}}{d \Omega^{k-1}} .
$$

The dimension of the de Rham cohomology, called the Betti number, is a basic topological invariant of smooth manifolds. For manifolds with an additional symplectic structure, the study of their differential forms have recently led to the discovery of new geometrical invariants. Specifically, Tseng and Yau ${ }^{9,10}$ recently introduced new cohomologies of differential forms on symplectic manifolds. These cohomologies differ from de Rham as they are non-topological and can vary with the symplectic structure on the manifold. Hence it is an interesting question to ask what these new symplectic invariants can tell us about the phase space.

In Section II, we shall motivate and review the construction of the special cohomologies of differential forms on symplectic manifolds. We will provide the physical motivation behind the existence of such symplectic cohomologies and give some basic mathematical backgrounds. In Section III, we shall explicitly calculate the symplectic cohomologies on the cotangent bundle and make comparison with the de Rham cohomology. We shall see how our results differs from previous calculations of symplectic cohomologies on compact spaces. We will also consider an example where the dynamical system has a symmetry, which by Noether's theorem leads to an integral of motion and a reduction of the phase space by two dimensions. As the reduced phase space remains symplectic but is now a closed manifold, it is interesting to consider how the symplectic cohomologies change under such a reduction. 


\section{SYMPLECTIC COHOMOLOGIES}

In this section, we will discuss the cohomologies of differential forms on symplectic manifolds. We will first give the physical background for these cohomologies and describe how they arise in physics, in particular within type IIA string theory. We then proceed to define them mathematically.

\section{A. Cohomology of differential forms in physics}

Heuristically, one can motivate the existence of a distinctive symplectic cohomology of differential forms directly from string theory ${ }^{11}$. To begin, let us first consider an example of how a cohomology can appear in physics by recalling the intrinsic relationship between de Rham cohomology and electromagnetism, in the context of Maxwell equations in four dimensions. On a four manifold $X^{4}=\mathbb{R}_{t} \times X^{3}$ with local coordinates $(t, \vec{x})$ and metric $g$, the Maxwell equations can be written concisely as

$$
\begin{gathered}
d F=0, \\
d^{*} F=* \rho_{e},
\end{gathered}
$$

where $F$ is the two-form field strength of the electromagnetic gauge field, $d^{*}$ is the standard metric dependent adjoint of the exterior derivative operator $d$, and $* \rho_{e}=\sum_{m} \delta\left(\vec{x}-\vec{x}_{m}\right) d t$ is the source term with delta functions centered at the spatial locations, $\vec{x}_{m}$, of electric point charges. Clearly, away from the location of electric charges, or mathematically outside the support $S$ of the charges, the delta functions is zero and so the field strength $F$ satisfies the conditions

$$
d F=0, \quad d^{*} F=0,
$$

which are precisely the harmonic conditions associated with the de Rham cohomology $H^{2}\left(X^{4} \backslash S\right)$. Additionally, if we would like to consider the solution space of $F$ for a fixed electric charge configuration, we can study the variation of the Maxwell equations, varying $F \rightarrow F+\delta F$ with $\delta \rho_{e}=0$. The conditions for $\delta F$ are then

$$
d \delta F=0, \quad d^{*} \delta F=0 .
$$

Therefore, $\delta F$ is parametrized by the harmonic two-forms of the de Rham cohomology, $\mathcal{H}^{2}\left(X^{4}\right)$. All in all, we see the close interrelations between the second de Rham cohomology 
and the Maxwell equations.

Now let us turn to string theory and its relation to cohomology of differential forms on symplectic manifolds. The relation involves type IIA string theory which is a theory that is naturally situated in ten dimensional spacetime. The ten-dimensional spacetime can often be taken simply to be a product manifold, $M^{3,1} \times X^{6}$, where $M^{3,1}$ is the four-dimensional Minkowski spacetime and $X^{6}$ is an six-dimensional internal manifold which for our purpose here will be taken to be a symplectic manifold.

Generally, an important and useful spacetime symmetry to preserve in string theory is supersymmetry. The requirement of supersymmetry necessarily imposes certain geometrical conditions on $X^{6}$. Indeed, supersymmetry requires that $X^{6}$ be a symplectic manifold with an $S U(3)$ structure, that is, it contains a no-where vanishing $(3,0)$-form, $\Omega$, which however need not be $d$-closed. ${ }^{2,3}$ However, $\Omega$ must satisfy the following conditions ${ }^{7,11}$

$$
\begin{gathered}
d \operatorname{Re} \Omega=0, \\
\left(d d^{\Lambda}\right)^{*} \operatorname{Re} \Omega=* e^{2 f} \rho_{A},
\end{gathered}
$$

where $d^{\Lambda}=d \Lambda-\Lambda d$ is the symplectic adjoint operator (where the operation $\Lambda$ is defined as the interior product with $\left.\omega^{-1}\right), * \rho_{A}$ is the three-form source term of three-dimensional membranes, and $e^{2 f}$ is an additional conformal factor. (These membranes, specifically O6and D6-branes are roughly generalization of "magnetic" point charges of electromagnetism. ${ }^{6}$ ) The conditions (II.3)-(II.4) motivate a comparison with the Maxwell equations (II.1)-(II.2), especially if one makes the following identifications:

$$
\operatorname{Re} \Omega \rightarrow F, \quad e^{2 f} \rho_{A} \rightarrow \rho_{e}, \quad\left(d d^{\Lambda}\right)^{*} \rightarrow d^{*}
$$

Carrying through with the Maxwell analogy at the cohomological level, the string equations intrinsically are suggestive of the following "harmonic conditions" for the three-form $\operatorname{Re} \Omega$,

$$
d \operatorname{Re} \Omega=0, \quad\left(d d^{\Lambda}\right)^{*} \operatorname{Re} \Omega=0
$$

Alternatively, if we note the relation of the Hodge star operator, $* \operatorname{Re} \Omega=\operatorname{Im} \Omega$, then (II.5) can be equivalently expressed as

$$
d d^{\Lambda} \operatorname{Im} \Omega=0, \quad d^{*} \operatorname{Im} \Omega=0 .
$$

The question then is whether there are cohomologies on symplectic manifolds whose harmonic conditions correspond to (II.5) and (II.6)? Indeed, they are precisely the harmonic 
conditions for the symplectic cohomologies

$$
\begin{gathered}
P H_{d+d^{\Lambda}}^{3}(X)=\frac{\left\{B \in \mathcal{P}^{3}(X) \mid d B=0\right\}}{d d^{\Lambda} \mathcal{P}^{3}(X)}, \\
P H_{d d^{\Lambda}}^{3}(X)=\frac{\left\{B \in \mathcal{P}^{3}(X) \mid d d^{\Lambda} B=0\right\}}{d \mathcal{P}^{2}(X) \cap \mathcal{P}^{3}(X)},
\end{gathered}
$$

where $\mathcal{P}^{3}(X)$ is the space of primitive three-forms on $X$. (The notion of a primitive form will be defined in the next subsection.) In fact, these cohomologies analogous to the Maxwell case also have a role in determining the local space of solutions of the above symplectic type IIA equations (II.3)-(II.4). ${ }^{11}$

Having seen that type IIA string theory suggests certain distinctive symplectic cohomologies of differential forms, let us now give a more rigorous mathematical construction of symplectic cohomologies.

\section{B. Linear differential symplectic operators and symplectic cohomologies}

To describe the symplectic cohomologies of differential forms, it is useful to start first with a discussion of the differential operators that are present. Considering first-order linear operators, the presence of a symplectic structure can decompose the exterior derivative $d$ into two distinct linear operators, just like the presence of a complex structure on a complex manifold decomposes $d$ into two Dolbeault operators $(\partial, \bar{\partial})$.

Let us recall first how this works in the complex case. On a complex manifold, every local neighborhood has a set of holomorphic and anti-holomorphic coordinates, $\left\{z_{i}, \bar{z}_{i}\right\}$. Hence, the space of differential form of a fixed degree $k, \Omega^{k}$ can be decomposed into $(p, q)$ components, $\mathcal{A}^{p, q}$ with $p d z_{i}$ 's and $q d \bar{z}$ 's, i.e.

$$
\Omega^{k}=\bigoplus_{p+q=k} \mathcal{A}^{p, q}
$$

The exterior derivative acting on each component gives two terms:

$$
d: \mathcal{A}^{p, q} \rightarrow \mathcal{A}^{p+1, q} \oplus \mathcal{A}^{p, q+1}
$$

The Dolbeault operators $\partial$ and $\bar{\partial}$ are then defined simply as the projections of $d \mathcal{A}^{p, q}$ onto $\mathcal{A}^{p+1, q}$ and $\mathcal{A}^{p, q+1}$, respectively. And hence, we have

$$
d=\partial+\bar{\partial}=d z_{i} \wedge \otimes \frac{\partial}{\partial z_{i}}+d \bar{z}_{i} \wedge \otimes \frac{\partial}{\partial \bar{z}_{i}} .
$$


In the symplectic case, there is also a natural decomposition of $d$ into two linear differential operators written as $\left(\partial_{+}, \partial_{-}\right) .{ }^{10}$ Indeed, we can imitate the above construction of the Dolbeault operators. First, the presence of a symplectic form leads to a decomposition of differential forms, but now into representations of the $\mathfrak{s l}(2)$ lie algebra. The generators of $\mathfrak{s l}(2)$ algebra constitute three natural actions on the space of differential forms in the presence of a symplectic form $\omega$ :

$$
\begin{aligned}
L: & A \rightarrow \omega \wedge A \\
\Lambda: & A \rightarrow \frac{1}{2}\left(\omega^{-1}\right)^{i j} i_{\partial_{x^{i}}} i_{\partial_{x j}} A \\
H: & A \rightarrow(n-k) A \quad \text { for } A \in \Omega^{k}(M)
\end{aligned}
$$

where the first is just the exterior product, the second is the interior product with respect to $\omega^{-1}$, and the third is simply the multiplication of the degree of the differential form up to a normalization with the dimension of the manifold $d=2 n$. These three generators have the following commutation relations:

$$
[H, \Lambda]=2 \Lambda, \quad[H, L]=-2 L, \quad[\Lambda, L]=H
$$

These commutations are in fact identical to the standard angular momentum commutation relations well-known to physicists:

$$
\left[J_{z}, J_{+}\right]=J_{+} \quad\left[J_{z}, J_{-}\right]=-J_{-} \quad\left[J_{+}, J_{-}\right]=2 J_{z}
$$

with the identifications $(\Lambda, L, H) \sim\left(J_{+}, J_{-}, 2 J_{z}\right)$.

Therefore, just as the angular momentum eigenstates in quantum mechanics are arranged into finite-dimensional representation of $\mathfrak{s l}(2)^{5}$, so likewise is the space of differential forms on a symplectic manifold. For angular momentum, an irreducible representation consists of eigenstates labelled by $\mid j, m>$ and starts with the highest weight $\mid j, m=j>$ and proceeds with the lowering operator $J_{-}$until one reaches $\mid j, m=-j>$ :

$$
\left\{\left|j, j>, J_{-}\right| j, j>, \ldots,\left(J_{-}\right)^{2 j} \mid j, j>\right\}
$$

Similarly, for forms, with $H \sim 2 J_{z}$, the highest weight are called primitive forms, whose space we denote by $\mathcal{P}(M)$. A differential $s$-form is primitive, i.e. $B_{s} \in \mathcal{P}^{s}(M)$, if

$$
\Lambda B_{s}=0 \quad \text { or equivalently, } L^{n-s+1} B_{s}=0
$$


which correspond to the conditions $J_{+} \mid j, j>=0$ and $\left(J_{-}\right)^{2 j+1} \mid j, j>=0$, respectively. Thus, each irreducible $\mathfrak{s l}(2)$ module (the mathematical term for what physicists call a representation) consists of the elements

$$
\left\{B_{s}, \omega \wedge B_{s}, \omega^{2} \wedge B_{s}, \ldots, \omega^{n-s} \wedge B_{s}\right\}
$$

Now since each element of the above basis element is composed of $\omega$ raised to some power $r$ exterior multiplied with a primitive $s$-form $B_{s}$, it is natural to label a basis element of an $\mathfrak{s l}(2)$ representation by the pair $(r, s)$ and define

$$
\mathcal{L}^{r, s}(M)=\left\{A \in \Omega^{2 r+s}(M) \mid A=\omega^{r} \wedge B_{s} \text { and } \Lambda B_{s}=0\right\}
$$

This gives us a decomposition of a differential $k$-form as

$$
\Omega^{k}=\bigoplus_{2 r+s=k} \mathcal{L}^{r, s}
$$

Comparing (II.10) with (II.9), these $\mathcal{L}^{r, s}$ are in a rough sense the symplectic analogs of $\mathcal{A}^{p, q}$ of complex geometry.

Carrying through the analogy with the complex case, we can proceed to act on $\mathcal{L}^{r, s}$ by the exterior derivative $d$. Since $d \omega=0$, we have

$$
d \mathcal{L}^{r, s}=d\left(\omega^{r} \wedge B_{s}\right)=\omega^{r} \wedge d B_{s} .
$$

Now for $d$ acting on a primitive form, it can be shown (see for example ${ }^{4}$ ) that

$$
d B_{s}=B_{s+1}^{0}+\omega \wedge B_{s-1}^{1} .
$$

Combining the above two equations, we find that

$$
d: \mathcal{L}^{r, s} \rightarrow \mathcal{L}^{r, s+1} \oplus \omega \wedge \mathcal{L}^{r, s-1}
$$

which has only just two components on the right hand side just as in the complex case. Now projecting onto each component, we can express the exterior derivative as ${ }^{10}$

$$
d \quad=\partial_{+} \quad+\omega \wedge \partial_{-}
$$

where the first-order differential operators $\left(\partial_{+}, \partial_{-}\right)$are defined by the derivative mapping

$$
\begin{aligned}
& \partial_{ \pm}: \mathcal{L}^{r, s} \longrightarrow \mathcal{L}^{r, s \pm 1} \\
& \partial_{ \pm}: \mathcal{P}^{s} \longrightarrow \mathcal{P}^{s \pm 1} \quad \text { for } r=0
\end{aligned}
$$


By the above definitions, $\partial_{+}$and $\partial_{-}$, respectively, raise and decrease the degree of the forms by one. Moreover, $\left(\partial_{+}, \partial_{-}\right)$are operators that map primitive forms to primitive forms (in the case of $r=0)$. And as with their complex counterparts, it follows from $d^{2}=0$ and the Lefschetz decomposition that they square to zero, i.e.

$$
\left(\partial_{+}\right)^{2}=\left(\partial_{-}\right)^{2}=0
$$

and anticommute: $\omega \wedge\left(\partial_{+} \partial_{-}\right)=-\omega \wedge\left(\partial_{-} \partial_{+}\right)$.

With the linear symplectic operators, $\left(\partial_{+}, \partial_{-}\right)$at hand we can now write down new primitive symplectic cohomologies ${ }^{9,10}$ as given in Table I below.

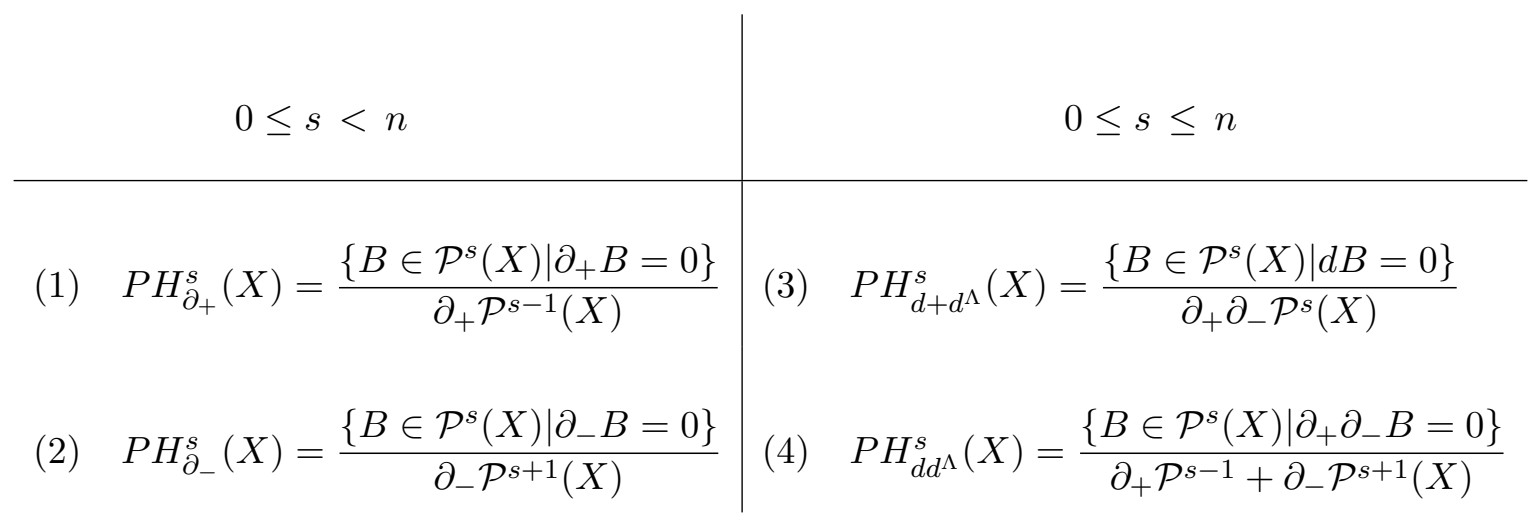

TABLE I. Primitive symplectic cohomologies.

The above symplectic cohomologies were shown to be all finite-dimensional on compact symplectic manifolds and have interesting properties. Some of the basic properties of these new cohomologies have already been analyzed..$^{8-10}$ Since they are associated with an elliptic complex, each has an associated elliptic Laplacian and thus have the standard desirable Hodge theoretical properties. Moreover, the cohomologies have been explicitly calculated for some non-Kähler symplectic nilmanifolds and indeed these cohomologies lead to new symplectic invariants. Perhaps not too surprisingly, the new invariants do not contain new information when the manifold is Kähler.

In the next section, we shall consider the case of these cohomologies on the non-compact phase space, i.e. the cotangent bundle $T^{*} M$.

\section{COHOMOLOGIES ON COTANGENT BUNDLE}

We now calculate the symplectic cohomologies on the cotangent bundle $X=T^{*} M$ and make comparison with the de Rham cohomology. To start, we consider the simplest case 


$$
\begin{aligned}
& H_{d R}^{k}\left(\mathbb{R}^{2 n}\right)= \begin{cases}\mathbb{R} & \text { if } k=0 \\
0 & \text { if } 1 \leq k \leq 2 n\end{cases} \\
& P H_{\partial_{+}}^{k}\left(\mathbb{R}^{2 n}\right)= \begin{cases}\mathbb{R} & \text { if } k=0 \\
\mathbb{R} & \text { if } k=1 \\
0 & \text { if } 2 \leq k<n\end{cases} \\
& P H_{\partial_{-}}^{k}\left(\mathbb{R}^{2 n}\right)=\quad 0 \quad \text { if } 0 \leq k<n \\
& P H_{d+d^{\Lambda}}^{k}\left(\mathbb{R}^{2 n}\right)=\left\{\begin{aligned}
\mathbb{R} & \text { if } k=0 \\
0 & \text { if } 1 \leq k \leq n
\end{aligned}\right. \\
& P H_{d d^{\Lambda}}^{k}\left(\mathbb{R}^{2 n}\right)= \begin{cases}0 & \text { if } k=0 \\
\mathbb{R} & \text { if } k=1 \\
0 & \text { if } 2 \leq k \leq n\end{cases}
\end{aligned}
$$

TABLE II. De Rham and symplectic cohomologies for $\mathbb{R}^{2 n}$. The symplectic cohomologies results follow from various Poincaré lemmas. ${ }^{10}$

of a particle moving in Euclidean space, $\mathbb{R}^{n}$. The cotangent bundle is simply $X=\mathbb{R}^{2 n}$. Taking as coordinates $\left\{x_{1}, \ldots, x_{n}, x_{n+1}, \ldots, x_{2 n}\right\}=\left\{x_{1}, \ldots, x_{n}, p^{1}, \ldots, p^{n}\right\}$ and the canonical symplectic form $\omega=\sum d x_{i} \wedge d x_{n+i}$, the known Poincaré lemmas ${ }^{10}$ give us the results which we list in Table II.

Note that with the exception of $P H_{d+d^{\Lambda}}^{k}\left(\mathbb{R}^{2 n}\right)$, the other symplectic cohomologies on $\mathbb{R}^{2 n}$ differ distinctively from that of de Rham $H_{d R}^{k}\left(\mathbb{R}^{2 n}\right)$. The differences arise due to the symplectic form $\omega=-d \alpha$ being $d$-exact and hence trivial in de Rham cohomology. However, the tautological one-form $\alpha$, which is not $d$-closed, is $\partial_{+}$-closed, i.e. $\partial_{+} \alpha=0$, but not $\partial_{+}{ }^{-}$ exact. Hence, $\alpha \in P H_{\partial_{+}}^{1}\left(\mathbb{R}^{2 n}\right)$ and it is also non-trivial in $P H_{d d^{\Lambda}}^{1}\left(\mathbb{R}^{2 n}\right)$.

We proceed now to the general cotangent bundle case $X=T^{*} M$ with base $M$ being any oriented manifold without boundary. Due to the fact that $M$ is a deformation retract of $X$ and that the de Rham cohomology is homotopically invariant, we have

$$
H_{d R}^{k}(X)=H_{d R}^{k}(M)
$$

So in the de Rham case, all the cohomological data on the bundle $X$ comes from the base $M$. 
For the symplectic cohomologies, based on the results for $X=\mathbb{R}^{2 n}$ case, we expect that they should contain more information, for instance, at least involving the tautological one-form. With a local coordinate chart $\left\{x_{1}, \ldots, x_{n}, x_{n+1}, \ldots, x_{2 n}\right\}$ and the canonical symplectic form $\omega=-d \alpha=\sum d x_{i} \wedge d x_{n+i}$, we find the following:

Proposition III.1 The primitive symplectic cohomologies of the cotangent bundle $X=$ $T^{*} M$ with respect to the canonical symplectic form are

1. $P H_{\partial_{+}}^{0}(X)=H_{d R}^{0}(M)$ and $P H_{\partial_{+}}^{k}(X)=\left\{H_{d R}^{k}(M), \alpha \wedge H_{d R}^{k-1}(M)\right\}$ for $1 \leq k<n$;

2. $P H_{\partial_{-}}^{k}(X)=0$ for $0 \leq k<n$;

3. $P H_{d+d^{\Lambda}}^{k}(X)=H_{d R}^{k}(M)$ for $0 \leq k \leq n$;

4. $P H_{d d^{\Lambda}}^{0}(X)=0, P H_{d d^{\Lambda}}^{k}(X)=\left\{\alpha \wedge H_{d R}^{k-1}(M)\right\}$ for $1 \leq k<n$ and $P H_{d d^{\Lambda}}^{n}(X)=$ $\left\{H_{d R}^{n}(M), \alpha \wedge H_{d R}^{n-1}(M)\right\}$.

Proof. Let $\pi: X \rightarrow M$. Observe first that any $k$-form which is pulled-back from $M$ is an element in $\mathcal{P}^{k}(X)$. That is, if $\eta_{k} \in \Omega^{k}(M)$, then clearly $\Lambda\left(\pi^{*} \eta_{k}\right)=0$ and $\pi^{*} \eta_{k} \in \mathcal{P}^{k}(X)$. As it should be clear when we are referring to forms on $X$ which are pull-backed from $M$, we will drop the $\pi^{*}$ notation when writing these forms.

(For $\left.P H_{\partial_{-}}^{k}(X)\right)$ It is clear that $P H_{\partial_{-}}^{0}(X)=H_{d R}^{2 n}(X)=0$. We compute the others by induction. Suppose that $P H_{\partial_{-}}^{i}(X)=0$ for all $i<k-1$. Let $B_{k} \in \mathcal{P}^{k}(X)$ be $\partial_{-}$-closed. It follows that $\omega^{n-k} B_{k}$ is $d$-closed. Since $H_{d R}^{2 n-k}(X)=0, \omega^{n-k} B_{k}=d A_{2 n-k-1}$ for some $A_{2 n-k-1} \in \Omega^{2 n-k-1}(X)$.

We now explain that $A_{2 n-k-1}$ can be chosen to be in $L^{n-k-1} \mathcal{P}^{k+1}$. Write $A_{2 n-k-1}$ as $\sum_{l=0}^{j} \omega^{n-k-1+l} B_{k+1-2 l}^{\prime}$. If $j>0$, it follows from $\omega^{n-k} B_{k}=d A_{2 n-k-1}$ that $\partial_{-} B_{k+1-2 j}^{\prime}=0$. By the induction hypothesis, there exists a $B_{k+2-2 j}^{\prime} \in \mathcal{P}^{k+2-2 j}(X)$ such that $\partial_{-} B_{k+2-2 j}^{\prime}=$ $B_{k+1-2 j}^{\prime}$. Let $A_{2 n-k-1}^{\prime}=A_{2 n-k-1}-\omega^{n-k-2+j} d B_{k+2-2 j}^{\prime}$. The exterior derivative of $A_{2 n-k-1}^{\prime}$ is still $\omega^{n-k} B_{k}$, and it has less components than $A_{2 n-k-1}$. By performing the procedure consecutively, we can make $A_{2 n-k-1}$ to be in $L^{n-k-1} \mathcal{P}^{k+1}$. It follows that $B_{k}$ is $\partial_{-}$-exact.

$\left(\right.$ For $\left.P H_{d+d^{\Lambda}}^{k}(X)\right)$ Let $B_{k} \in \mathcal{P}^{k}(X)$ be $d$-closed. Since $H_{d R}^{k}(X)=H_{d R}^{k}(M)$, there exist a $\left[\xi_{k}\right] \in H_{d R}^{k}(M)$ and a $A_{k-1}^{\prime} \in \Omega^{k-1}(X)$ such that $B_{k}=\xi_{k}+d A_{k-1}^{\prime}$. By the same argument used in $P H_{\partial_{-}}^{k}(X), A_{k-1}^{\prime}$ can be replaced by a $B_{k-1}^{\prime} \in \mathcal{P}^{k-1}(X)$, and we have $B_{k}=\xi_{k}+d B_{k-1}^{\prime}$. 
The non-primitive component reads $\partial_{-} B_{k-1}^{\prime}=0$. From $P H_{\partial_{-}}^{k-1}(X)=0$, there exists a $B_{k}^{\prime} \in \mathcal{P}^{k}$ such that $B_{k-1}^{\prime}=\partial_{-} B_{k}^{\prime}$. Therefore, $B_{k}=\xi_{k}+\partial_{+} \partial_{-} B_{k}^{\prime}$.

On the other hand, $\partial_{+} \partial_{-}$-exactness implies $d$-exactness. It follows that any nontrivial element in $H_{d R}^{k}(X)=H_{d R}^{k}(M)$ cannot be $\partial_{+} \partial_{-}$-exact.

(For $P H_{d d^{\Lambda}}^{k}(X)$ for $\left.0 \leq k<n\right)$ It is clear that $P H_{d d^{\Lambda}}^{0}(X)=0$. For any $1 \leq k<n$, suppose that $B_{k} \in \mathcal{P}$ is $\partial_{+} \partial_{-}$-closed. Then $\partial_{-} B_{k} \in \mathcal{P}^{k-1}$ is $d$-closed. Since $P H_{d+d^{\Lambda}}^{k-1}(X)=$ $H_{d R}^{k-1}(M)$, there exist a $\left[\xi_{k-1}\right] \in H_{d R}^{k-1}(M)$ and a $B_{k-1}^{\prime} \in \mathcal{P}^{k-1}$ such that $\partial_{-} B_{k}=\xi_{k-1}+$ $\partial_{+} \partial_{-} B_{k-1}^{\prime}$. It follows that $B_{k}-\alpha \wedge \xi_{k-1}+\partial_{+} B_{k-1}^{\prime}$ is $\partial_{-}$-closed. Note that $\alpha \wedge \xi_{k-1} \in \mathcal{P}^{k}$. Since $P H_{\partial_{-}}^{k}(X)=0$, there exists a $B_{k+1}^{\prime} \in \mathcal{P}^{k+1}$ such that $B_{k}=\alpha \wedge \xi_{k-1}-\partial_{+} B_{k-1}^{\prime}+\partial_{-} B_{k+1}^{\prime}$.

If $\alpha \wedge \xi_{k-1}$ belongs to $\partial_{+} \mathcal{P}^{k-1}+\partial_{-} \mathcal{P}^{k+1}$, then $\partial_{-}\left(\alpha \wedge \xi_{k-1}\right)=\xi_{k-1}$ is $\partial_{+} \partial_{-}$-exact. It follows that $\xi_{k-1}$ is $d$-exact.

(For $\left.P H_{d d^{\Lambda}}^{n}(X)\right)$ Suppose that $B_{n} \in \mathcal{P}^{n}$ is $\partial_{+} \partial_{-}$-closed. The same argument as above finds a $\left[\xi_{n-1}\right] \in H_{d R}^{n-1}(M)$ and a $B_{n-1}^{\prime} \in \mathcal{P}^{n-1}$ such that $B_{n}-\alpha \wedge \xi_{n-1}+\partial_{+} B_{n-1}^{\prime}$ is $\partial_{-}$-closed. In the middle dimension, being $\partial_{-}$-closed is the same as being $d$-closed. Therefore, there exist a $\left[\xi_{n}\right] \in H_{d R}^{n}(M)$ and an $A_{n-1} \in \Omega^{n-1}(X)$ such that $B_{n}-\alpha \wedge \xi_{n-1}+\partial_{+} B_{n-1}^{\prime}=\xi_{n}+d A_{n-1}$. By the same trick as that used in $P H_{\partial_{-}}^{k}(X), A_{n-1}$ can be replaced by a $B_{n-1}^{\prime \prime} \in \mathcal{P}^{n-1}(X)$. Then we have $B_{n}=\alpha \wedge \xi_{n-1}+\xi_{n}-\partial_{+} B_{n-1}^{\prime}+d B_{n-1}^{\prime \prime}$. The primitive component reads $B_{n}=\alpha \wedge \xi_{n-1}+\xi_{n}+\partial_{+}\left(B_{n-1}^{\prime \prime}-B_{n-1}^{\prime}\right)$.

Suppose that $\alpha \wedge \xi_{n-1}+\xi_{n}$ is $\partial_{+}$-exact. By taking $\partial_{-}, \xi_{n-1}$ is $\partial_{+} \partial_{-}$-exact, and must be $d$-exact. Thus, there exists a $\eta_{n-2} \in \Omega^{n-2}(M)$ such that $\xi_{n-1}=d \eta_{n-2}$. Then $\alpha \wedge \xi_{n-1}=$ $\alpha \wedge\left(d \eta_{n-2}\right)=-\partial_{+}\left(\alpha \wedge \eta_{n-2}\right)$. It follows that $\xi_{n}$ is $\partial_{+}$-exact. We write $\xi_{n}$ as $\partial_{+} \tilde{B}_{n-1}$ for a $\tilde{B}_{n-1} \in \mathcal{P}^{n-1}(X)$. Since $d \xi_{n}=0, \tilde{B}_{n-1}$ is necessarily $\partial_{+} \partial_{-}$-closed. With $\operatorname{PH}_{d d^{\Lambda}}^{n-1}(X)=$ $\alpha \wedge H_{d R}^{n-2}(M)$, there must exist a $\left[\tilde{\xi}_{n-2}\right] \in H_{d R}^{n-2}(M)$, a $\tilde{B}_{n-2} \in \mathcal{P}^{n-2}$ and a $\tilde{B}_{n} \in \mathcal{P}^{n}$ such that $\tilde{B}_{n-1}=\alpha \wedge \tilde{\xi}_{n-2}+\partial_{+} \tilde{B}_{n-2}+\partial_{-} \tilde{B}_{n}$. Taking $\partial_{+}$gives $\xi_{n}=\partial_{+} \partial_{-} \tilde{B}_{n-2}$, and $\xi_{n}$ must be $d$-exact.

(For $P H_{\partial_{+}}^{k}(X)$ ) It is clear that $P H_{\partial_{+}}^{0}(X)=H_{d R}^{0}(M)$. For any $1 \leq k<n$, suppose that $B_{k} \in \mathcal{P}^{k}(X)$ is $\partial_{+}$-closed. Then $d B_{k}=\omega \wedge B_{k-1}^{\prime}$ for some $B_{k-1}^{\prime} \in \mathcal{P}^{k-1}(X)$. It follows from $d^{2} B_{k}=0=\omega \wedge\left(d B_{k-1}^{\prime}\right)$ that $B_{k-1}^{\prime}$ is $d$-closed. Since $P H_{d+d^{\Lambda}}^{k-1}(X)=H_{d R}^{k-1}(M)$, there exist a $\left[\xi_{k-1}\right] \in H_{d R}^{k-1}(M)$ and a $B_{k-1}^{\prime \prime} \in \mathcal{P}^{k}(X)$ such that $B_{k-1}^{\prime}=\xi_{k-1}+\partial_{+} \partial_{-} B_{k-1}^{\prime \prime}$. It follows that $B_{k}-\alpha \wedge \xi_{k-1}+\partial_{+} B_{k-1}^{\prime \prime}$ is $d$-closed. The rest of the argument is essentially the same as that 
for $P H_{d d^{\Lambda}}^{n}(X)$.

Let us remark on these results.

Clearly, on the cotangent bundle $X=T^{*} M$, the four symplectic cohomologies calculated above are very different from de Rham cohomology. Nevertheless, the results we found can be expressed simply in terms of the de Rham cohomology of the base $M$ and the tautological one-form, $\alpha$. That such is the case can be expected. For the manifold $X$ is completely determined by the base $M$, which is in general just a smooth manifold without any additional structure assumed. The symplectic cohomologies thus contain the information of the differential forms on $M$ in terms of $H_{d R}^{k}(M)$ and additionally the information of the canonical symplectic structure of $T^{*} M$ which is given by the tautological one-form, $\alpha$. Of course, if one were to impose additional structure on $M$, for instance, if $M$ is also a symplectic manifold, then there may be other symplectic structures on $X$. In this case, the symplectic cohomologies with respect to these other non-canonical symplectic structures can lead to different results. This is in contrast with the de Rham cohomology which is purely topological and of course independent of the symplectic structure.

Notice also that the symplectic cohomologies on a non-compact space such as the cotangent bundle do not have the duality or isomorphism properties that are present on a compact space. Specifically, if $\left(N^{2 n}, \omega\right)$ is a compact symplectic manifold, then ${ }^{9,10}$

$$
P H_{\partial_{+}}^{k}(N) \cong P H_{\partial_{-}}^{k}(N), \quad \text { for } 0 \leq k<n
$$

and

$$
P H_{d+d^{\Lambda}}^{k}(N) \cong P H_{d d^{\Lambda}}^{k}(N), \quad \text { for } 0 \leq k \leq n
$$

Such relations clearly do not persist in the non-compact case, as the results here for the cotangent bundle demonstrate. However, as we have mentioned in Section II, the symplectic cohomologies are associated with an elliptic complex which has index zero. ${ }^{10}$ The symplectic cohomologies on non-compact manifold must still satisfy this zero index requirement. And indeed, this requirement can be easily checked to hold for the cotangent bundle.

Our emphasis here on the cotangent bundle has been motivated by phase space and Hamiltonian mechanics. But from a symplectic geometry point of view, the cotangent bundle is also an important basic object as it describes the tubular neighborhood of a lagrangian submanifold. Many standard constructions of symplectic manifolds today involve lagrangian 
surgeries. As such, our results here provide an important necessary ingredient for calculating the symplectic cohomologies using Mayer-Vietoris method ${ }^{8}$ for many constructions of symplectic manifolds.

Lastly, returning to the context of Hamiltonian mechanics, it is well-known that in the presence of a symmetry, Noether's theorem reduces the dimension of the phase space by two while preserving the symplectic structure. This process is known as the MarsdenWeinstein reduction or symplectic reduction ${ }^{1}$. Let us consider here the simplest case where $M=\mathbb{R}^{n}$ and $X=T^{*} M=\mathbb{R}^{2 n}$. We can write the standard symplectic form on $\mathbb{R}^{2 n}$ as $\omega=\sum d x_{i} \wedge d x_{n+1}=\sum r_{i} d r_{i} \wedge d \theta_{i}=\frac{i}{2} \sum d z_{i} \wedge d \bar{z}_{i}$, where the last expression is in terms of $\mathbb{C}^{n}$. Assume the dynamical system is symmetrical under the $S^{1}$-action on $\left(\mathbb{C}^{n}, \omega\right)$ given by

$$
\phi_{t}:\left(z_{1}, z_{2}, \ldots, z_{n}\right) \rightarrow e^{i t}\left(z_{1}, z_{2}, \ldots, z_{n}\right)
$$

where $t \in S^{1}$. Here, $\phi_{t}$ generates the vector field

$$
\xi=\frac{\partial}{\partial \theta_{1}}+\frac{\partial}{\partial \theta_{2}}+\ldots+\frac{\partial}{\partial \theta_{n}}
$$

and is also a hamiltonian action with moment map given by the function $\mu=-\frac{1}{2}|z|^{2}+\frac{1}{2}$. (For simplicity, we have set the constant to be $1 / 2$.) Clearly, we have,

$$
i_{\xi} \omega=d \mu
$$

Because of symmetry, the value of $\mu$ remains constant under dynamical motion. So the particle can be constrained to a level set, $\mu^{-1}(0)=S^{2 n-1}$. The orbit space is then

$$
\frac{\mu^{-1}(0)}{S^{1}}=\frac{S^{2 n-1}}{S^{1}}=\mathbb{C P}^{n-1}
$$

The symplectic cohomologies on this closed Kähler manifold $\mathbb{C P}^{n-1}$ can be easily calculated. For all four symplectic cohomologies, the only non-trivial class on $\mathbb{C P}^{n-1}$ is the degree zero cohomology (i.e. $P H_{\partial_{+}}^{0}, P H_{\partial_{-}}^{0}, P H_{d+d^{\Lambda}}^{0}, P H_{d d^{\Lambda}}^{0}$ ) generated by the constant function; all other degrees are trivial. Comparing this to the cohomologies of the original manifold $\mathbb{R}^{2 n}$ listed in Table II, we see that except for $P H_{d+d^{\Lambda}}^{k}$, the three other primitive symplectic cohomologies changed non-trivially under symplectic reduction. We leave it as an interesting open question to characterize how symplectic cohomologies change under a general symplectic reduction. 


\section{ACKNOWLEDGMENTS}

The work of S.-T. Yau is supported in part by NSF grants 0804454, 0854971, and 0937443.

\section{REFERENCES}

${ }^{1}$ A. Cannas da Silva, Lectures on Symplectic Geometry, Springer-Verlag, Berlin (2001).

${ }^{2}$ M. Grana, R. Minasian, M. Petrini, and A. Tomasiello, "Supersymmetric backgrounds from generalized Calabi-Yau manifolds," J. High Energy Phys. no. 08, 046 (2004).

${ }^{3}$ M. Grana, R. Minasian, M. Petrini, and A. Tomasiello, "Generalized structures of $N=1$ vacua," J. High Energy Phys. no. 11, 020 (2005).

${ }^{4}$ D. Huybrechts, Complex Geometry: An Introduction, Springer-Verlag, Berlin (2005).

${ }^{5}$ R. Liboff, Introductory Quantum Mechanics, Addison-Wesley, Boston (1992).

${ }^{6}$ J. Polchinski, String Theory, Vol. 2: Superstring Theory and Beyond, Cambridge University Press, New York (1998).

${ }^{7}$ A. Tomasiello, "Reformulating supersymmetry with a generalized Dolbeault operator," J. High Energy Phys. no. 2, 010 (2008).

${ }^{8}$ C.-J. Tsai, L.-S. Tseng and S.-T. Yau, work in preparation.

${ }^{9}$ L.-S. Tseng and S.-T. Yau, "Cohomology and Hodge theory on symplectic manifolds, I," Preprint: arXiv:0909.5418.

${ }^{10}$ L.-S. Tseng and S.-T. Yau, "Cohomology and Hodge theory on symplectic manifolds, II," Preprint: arXiv:1011.1250.

${ }^{11}$ L.-S. Tseng and S.-T. Yau, "Generalized cohomologies and supersymmetry," Preprint: arXiv:1111.6968. 